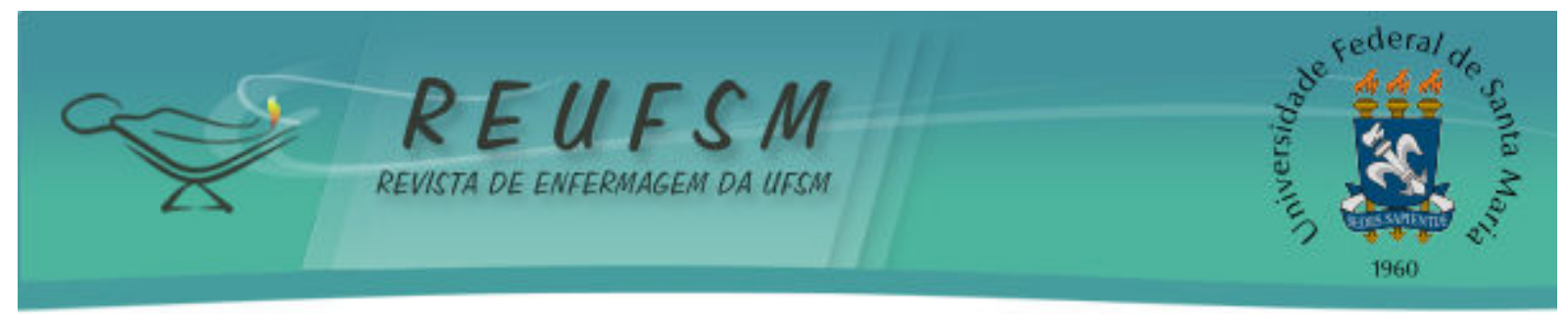

ARTIGO ORIGINAL

\title{
PERCEPÇÃO DA ENFERMAGEM SOBRE OS FATORES DE RISCO QUE ENVOLVEM A SEGURANÇA DO PACIENTE PEDIÁTRICO
}

\author{
PERCEPTION OF NURSING ON THE RISK FACTORS RELATED TO THE PEDIATRIC PATIENT \\ SAFETY
}

\section{PERCEPCIÓN DE ENFERMERÍA SOBRE LOS FACTORES DE RIESGO RELACIONADOS CON LA SEGURIDAD DEL PACIENTE PEDIATRICO}

Francine Terezinha de Souza ${ }^{1}$ Mayara Costa Garcia² Pâmella Priscilla da Silva Rangel ${ }^{3}$ Patrícia Kuerten Rocha ${ }^{4}$

\author{
Doi: $10.5902 / 217976928781$
}

RESUMO: Objetivo: conhecer a percepção da equipe de enfermagem sobre os fatores de risco e os métodos de barreira que podem interferir na segurança do paciente pediátrico durante sua hospitalização. Método: estudo de natureza qualitativa, realizado entre setembro e outubro de 2012 em um Hospital Universitário, por meio de entrevista semiestruturada com 18 profissionais de enfermagem. Os dados foram analisados utilizando-se o Modo Operativo, seguindo as seguintes etapas: pré-análise, exploração do material, tratamento dos resultados obtidos e interpretação. Resultados: as categorias encontradas, mais citadas durante as entrevistas, foram: definições de fatores de risco, terapia medicamentosa, identificação do paciente e métodos de barreira. Conclusão: fica evidente que os membros da equipe de enfermagem convergem em suas percepções, expressando situações do cotidiano como fatores de risco, além de citarem possíveis métodos de barreiras como, por exemplo, melhorar a identificação do paciente.

Descritores: Fatores de risco; Segurança do paciente; Enfermagem pediátrica.

ABSTRACT: Aim: to know the perception of the nursing staff about the risk factors and the barrier methods that may affect the safety of pediatric patients during their hospitalization. Method: qualitative study, conducted between September and October 2012 in an University Hospital, through semi-structured interviews with 18 nursing staff. Data were analyzed using the Operation Mode, the following steps: Pre-analysis, material exploration, processing and interpretation of results. Results: the categories found, most cited during the interviews were: definitions of risk factors, drug therapy, patient identification and barrier methods. Conclusion: it is evident that members of the nursing team converge in their perceptions, expressing everyday situations as risk factors, and possible methods of citing barriers as, for example, improve the patient's identification.

Descriptors: Risk factors; Patient safety; Pediatric nursing.

\footnotetext{
${ }^{1}$ Enfermeira, formada pela Universidade Federal de Santa Catarina, Florianópolis, Santa Catarina, Brasil. Email: francinetsouza@hotmail.com

2 Enfermeira, formada pela Universidade Federal de Santa Catarina, Florianópolis, Santa Catarina, Brasil. Email: maycgarcia@gmail.com

${ }^{3}$ Enfermeira, formada pela Universidade Federal de Santa Catarina, Florianópolis, Santa Catarina, Brasil. Email: panzinha_ph@hotmail.com

${ }^{4}$ Enfermeira. Doutora em Enfermagem pela Universidade Federal de Santa Catarina (UFSC). Professora Adjunto do Departamento de Enfermagem e do Programa de Pós-graduação em Enfermagem da Universidade Federal de Santa Catarina (UFSC). Florianópolis, Santa Catarina, Brasil. E-mail: pkrochaucip@gmail.com
} 


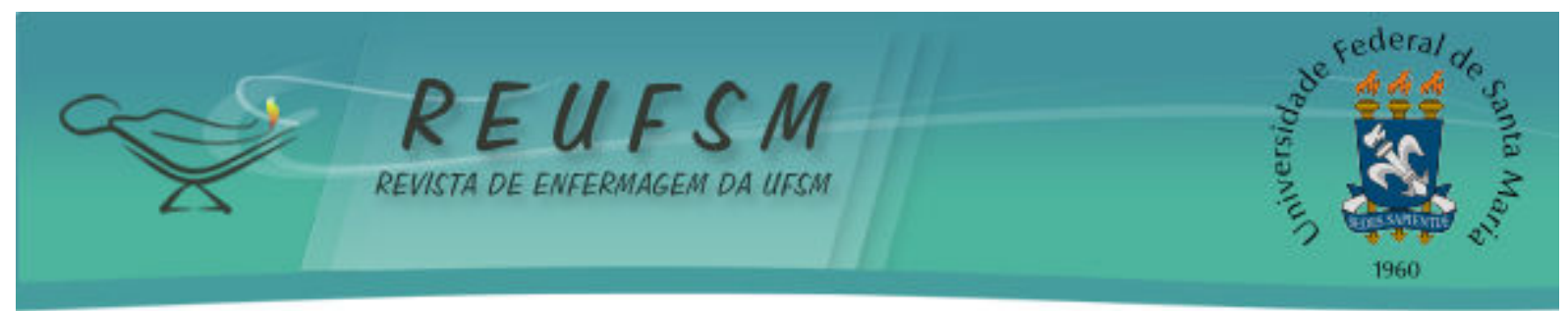

RESUMEN: Objetivo: conocer la percepción del equipo de enfermería sobre los factores de riesgo y los métodos de barrera que pueden afectar a la seguridad de los pacientes pediátricos durante su internación. Método: estudio cualitativo realizado entre septiembre y octubre de 2012 en un hospital universitario, por medio de entrevistas semiestructuradas con 18 profesionales de enfermería. Los datos fueron analizados utilizando el Modo Operativo, con las siguientes etapas: pre-análisis, exploración de materiales, procesamiento e interpretación de los resultados. Resultados: las categorías encontradas, más citados en las entrevistas fueron: la definición de los factores de riesgo, la terapia de drogas, la identificación del paciente y los métodos de barrera. Conclusión: es evidente que los miembros del equipo de enfermería convergen en sus percepciones, expresando situaciones cotidianas como factores de riesgo, además de citar posibles métodos de barreras como, por ejemplo, mejorar la identificación del paciente.

Descriptores: Factores de riesgo; Seguridad del paciente; Enfermería pediátrica.

\section{INTRODUÇÃO}

O ambiente hospitalar, com sua macrocomplexidade, oferece inúmeros riscos para a segurança do paciente, caracterizados pelos eventos adversos que podem resultar em efeitos negativos aos pacientes. ${ }^{1}$ Com isso a assistência ao paciente é constantemente ameaçada pelos fatores de risco, definidos como a probabilidade da ocorrência de um incidente durante a assistência em saúde. ${ }^{2}$

O tema Segurança do Paciente ganhou visibilidade após os Estados Unidos da América divulgarem, em 1999, resultados de saúde alarmantes. Em "To Err is Human" o país revelou que por lá ocorriam de 44.000 a 98.000 mortes anuais decorrentes de erros do sistema de saúde, e que as injúrias provocadas pelos eventos adversos preveníveis movimentavam entre 17 e 29 bilhões de dólares todos os anos. ${ }^{2}$

Desde então, muitos programas foram criados, tanto internacional quanto nacionalmente, com a finalidade de incentivar as pesquisas e sensibilizar os profissionais para o problema. O termo Segurança do Paciente pode ser definido como a redução do risco e de danos desnecessários associados à assistência em saúde, e é protegido e disseminado dentro da Cultura de Segurança, que é uma cultura de aprendizagem através das notificações dos resultados, onde a segurança de uma organização é prioridade. A organização deve focar em recolher o máximo de informação possível a respeito dos erros que permeiam seu desenvolvimento, sem expor, acusar ou punir quem erra, e sim fornecendo subsídios para que essas pessoas aprendam por meio dos erros. ${ }^{3}$

$\mathrm{Na}$ tentativa de estabelecer um ambiente seguro e evitar a ocorrência de erros, a Cultura de Segurança do Paciente preconiza que os incidentes ocorridos sejam relatados e notificados, possibilitando sua análise para que as instituições possam adotar práticas preventivas e educacionais fundamentadas. ${ }^{4}$ Para que a notificação do eventos ocorra, é recomendável que o método de punição frente ao erro seja abolido, uma vez que o medo perante a repressão dificulta que os trabalhadores assumam tais acontecimentos.

Estudo realizado na Inglaterra, entre 2007 e 2008, evidenciou a fragilidade que o público pediátrico possui frente à assistência em saúde. Frente ao objetivo de analisar os incidentes relacionados à medicação, identificou que a faixa etária mais acometida foi a de zero a quatro anos de idade, onde os erros de dose e/ou concentração corresponderam a $23 \%$ dos eventos envolvendo esta população. 0 segundo tipo de erro mais relatado foi o erro de omissão e o terceiro o erro de administração. ${ }^{5}$

Estudo realizado em uma Unidade de Cuidados Intensivos Pediátricos para atendimento de pacientes oncológicos, registrou 71 notificações de 110 erros de medicação, representando uma ocorrência de 227 erros por 1000 pacientes/dia. ${ }^{6}$ 


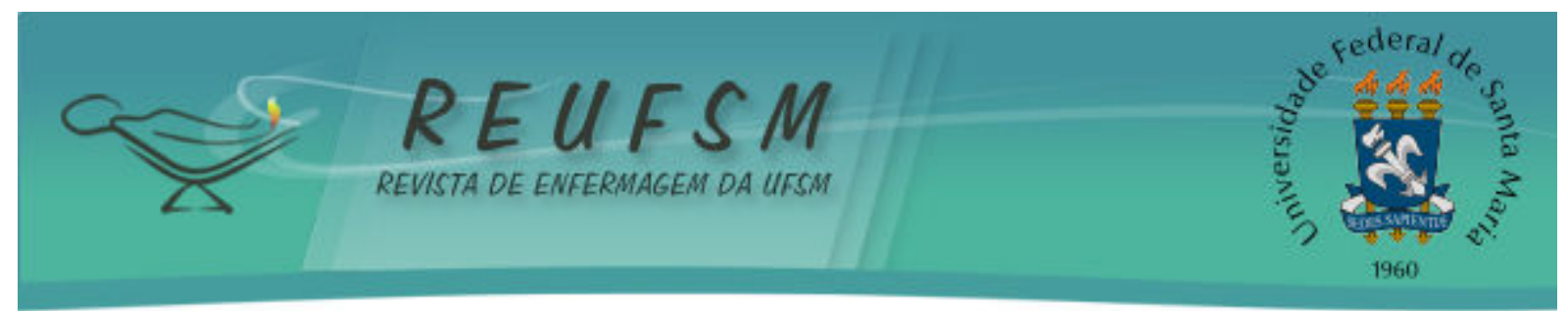

Nesse contexto, ressalta-se que a população pediátrica, possui risco de dano três vezes maior do que adultos na mesma situação. ${ }^{7}$ Quanto se trata de hospitalização infantil, muitos fatores estão envolvido para sua segurança, devido a suas especificidades quanto ao seu estágio de desenvolvimento, peso, idade, área corporal e condições clínicas. 0 zelo no cuidado com o paciente pediátrico exige dominar os erros e analisar quais fatores levaram a eles, a fim de implementar medidas de melhorarias que visem diminuí-los. ${ }^{8}$

Nesse sentido, existem os métodos de barreira que se caracterizam por ações que visam minimizar os fatores de risco que envolvem o processo de assistência em saúde, na tentativa de impedir a ocorrência de eventos adversos. Na literatura são exploradas como medidas preventivas dos erros e incidentes. ${ }^{9}$

Diante do exposto, a questão norteadora deste estudo foi: Qual a percepção da equipe de enfermagem quanto aos fatores de risco que podem interferir na segurança do paciente pediátrico durante o período de hospitalização em uma unidade de internação pediátrica de um Hospital Universitário da região sul do Brasil?

A partir disto, o estudo tem como objetivo conhecer a percepção da equipe de enfermagem sobre os fatores de risco e os métodos de barreira a serem implantados que podem interferir na segurança do paciente pediátrico durante sua hospitalização.

\section{MÉTODO}

Estudo de abordagem qualitativa, do tipo exploratório-descritiva, realizado com a equipe de Enfermagem de uma unidade de internação pediátrica de um Hospital Universitário da Região Sul do Brasil.

A coleta de dados ocorreu entre os meses de setembro e outubro de 2012, por meio de entrevista semi-estruturada, aplicada individualmente e no local de trabalho dos participantes, com agendamento prévio; sendo que as mesmas foram gravadas com o consentimento dos participantes.

A unidade de estudo contava, no período da coleta de dados, com uma equipe de oito enfermeiros, 26 técnicos de enfermagem e cinco auxiliares de enfermagem. Para inclusão dos participantes no estudo, seguiu-se critérios de inclusão e exclusão. Os critérios de inclusão foram: ter no mínimo seis meses de atuação no cenário da pesquisa e estar em exercício profissional no período da coleta. Os critérios de exclusão foram: estar afastado, em licença saúde ou férias no período do estudo. Seguindo estes critérios, participaram do estudo 18 profissionais da equipe de enfermagem.

Anteriormente à entrevista, foi realizado um pré-teste do questionário semiestruturado, a fim de averiguar o entendimento dos profissionais perante as questões. Para participação no pré-teste os profissionais foram escolhidos de maneira aleatória, sendo que participaram desta etapa duas enfermeiras e um técnico em enfermagem, que posteriormente, foram excluídos da amostra do estudo. A partir das considerações apontadas pelos participantes, o questionário foi ajustado.

Dessa maneira, a entrevista semi-estruturada realizada com os participantes do estudo compreendeu quatro questões: 0 que você entende por fatores de risco à segurança do paciente pediátrico? Quais os fatores de risco que você percebe na unidade? Quais geram intercorrência com maior frequência? 0 que você faria para que não existissem esses fatores de risco?

O anonimato do profissional foi assegurado e para identificar a categoria profissional foram utilizados códigos, da seguinte maneira: E para enfermeiros, T para técnicos e A para auxiliares de enfermagem; e, para identificar a entrevista foi acrescido o número da mesma. 


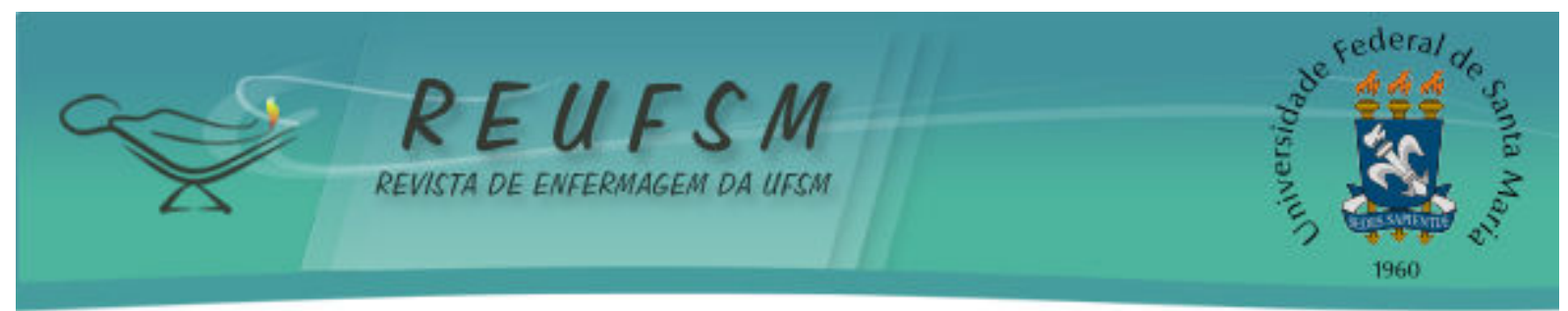

Após a transcrição das gravações, os dados obtidos por meio das entrevistas foram analisados de acordo com o Modo Operativo ${ }^{10}$, seguindo as seguintes etapas: pré-análise, exploração do material e tratamento dos resultados obtidos e interpretação.

0 projeto foi aprovado em 26 de Setembro de 2012 pelo Comitê de Ética da Universidade Federal de Santa Catarina, sob parecer $\mathrm{n}^{\circ} 108.551$, CAAE $\mathrm{n}^{\circ}$ 05232112.6.0000.0121. Durante a pesquisa, todos os preceitos éticos foram respeitados.

\section{RESULTADOS E DISCUSSÃO}

Participaram do estudo 18 profissionais da enfermagem, sendo seis enfermeiras, 11 técnicos de enfermagem e um auxiliar de enfermagem. Destes, 17 foram do sexo feminino e um do sexo masculino, e atuavam na referida unidade em média oito anos ( $\mathrm{dp} \pm$ 6,75 anos). Sete dos profissionais mantinham ainda, um segundo vínculo empregatício com outra instituição.

Após a análise e a categorização das informações levantadas, foram identificadas 14 categorias referentes às quatro questões propostas, que foram: definições de fatores de risco; procedimentos de enfermagem; identificação do paciente; terapia medicamentosa; competências profissionais; ambiente físico; transferência de informações frente aos pacientes e entre os profissionais; diagnóstico médico; imaturidade infantil; higienização das mãos e profissional como vetor; material permanente e tecnologia; rotatividade dos acompanhantes; déficits no número de profissionais; erro e métodos de barreiras. Sendo que destas, quatro serão exploradas neste artigo por contemplar o objetivo proposto e ganharem destaque referente às respostas dos profissionais pelo grande número de informações levantadas, que são: definição de fatores de risco, terapia medicamentosa, identificação do paciente e métodos de barreira.

\section{Definição de fatores de risco}

Os participantes tiveram diferentes formas de expressar o que entendiam sobre fatores de risco, havendo uma diferença na forma com que os mesmos responderam. Cinco, dos seis Enfermeiros, responderam com definições teóricas, formando conceitos. Oito dos doze técnicos de Enfermagem responderam com definições, enquanto os demais quatro técnicos responderam citando exemplos do cotidiano. Esta diferença se ilustra nas seguintes falas:

Eu acredito que seja tudo que pode comprometer a saúde da criança, que pode levar a uma falha. Alguma falha que acarrete no comprometimento na saúde da criança. (E. 5)

[...] está relacionada à atividade de enfermagem e de outras áreas também aqui da unidade, outras equipes de profissionais. Dentistas, médicos, psicólogos, até mesmo a limpeza, para mim cada um tem sua parcela [...]. (T. 12)

[...] fator de risco é tudo que põe em risco o cuidado, a saúde e a vida da criança. $E$ na pediatria, nós enquanto profissionais, desde o banho, medicação, brinquedo... tudo pode ser fator de risco [...]. (T. 7) 


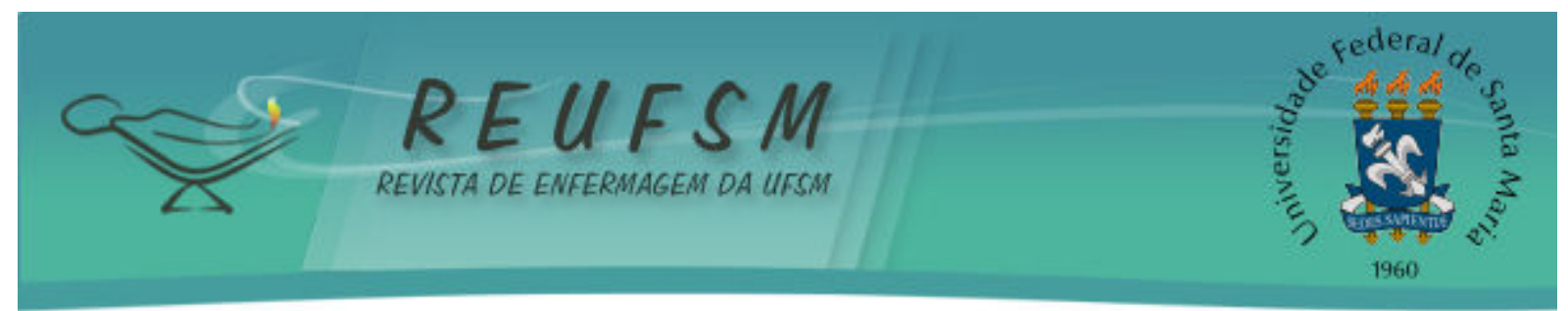

De acordo com a Organização Mundial da Saúde (OMS) conceitua-se fator de risco como a probabilidade da ocorrência de um incidente durante a assistência em saúde. ${ }^{3}$ Percebe-se que estes profissionais de enfermagem definem o significado de fatores de risco com as mesmas características da OMS, mas não realizam essa interligação.

Os profissionais ainda ressaltam que os fatores de risco estão presentes não só na Enfermagem, mas também nas outras áreas da saúde, assim como está presente no dia a dia hospitalar, ou seja, desde momentos como a realização de procedimentos complexos, ou até mesmo em situações corriqueiras como o simples brincar na unidade de pediatria.

De uma maneira geral, a literatura aponta que os principais fatores de risco à segurança do paciente abrangem diversas questões, como a falibilidade humana, problemas existentes no sistema de saúde, dispositivos e equipamentos inadequados para a assistência, comunicação ineficaz entre os membros da equipe, dimensionamento inadequado de pessoal, alta carga de trabalho e conhecimento restrito ou desatualizado sobre a segurança do paciente. ${ }^{11}$

Nesse sentido, os fatores que predispõe os erros podem ter origem relacionada aos profissionais, incluindo, por exemplo, as quedas do paciente, a infecção hospitalar, úlceras por pressão, e os erros de medicação; relacionados aos procedimentos, como um planejamento inadequado, ventilação artificial, manutenção de dispositivos como drenos e sondas; e ainda, relacionados ao sistema de saúde, os quais se caracterizam pelos fatores organizacionais, recursos de materiais e equipamentos. ${ }^{12}$

\section{Terapia medicamentosa}

A medicação foi o fator de risco que mais apareceu nas falas dos profissionais, onde cinco foram citadas nas falas de enfermeiros e dez nas falas de técnicos de enfermagem e ainda, uma na fala de auxiliar de enfermagem. Quanto aos fatores de risco na medicação, os seguintes itens foram mencionados: doses fracionadas, prescrição incorreta, as cinco certezas, manipulação, dispensação, cálculos de diluição, padronização da farmácia, uso coletivo, tempo de infusão e estabilidade.

Os participantes afirmaram que este é o fator de risco que possui a maior prevalência e o que mais gera erros na assistência ao paciente pediátrico. Foi mencionado este fator de risco que engloba todo o processo da medicação, ou seja, desde a apresentação das embalagens até $o$ ato de diluir e administrar o medicamento ao paciente, como pode se verificar nas seguintes falas:

Então eu acredito que aí é o nosso Tendão de Aquiles, questão da medicação, manipulação, preparo e administração. (E. 4)

Eu acho que a coisa da medicação, da diluição, a falta da padronização da farmácia é o que acontece mais frequente. (E. 5)

[...] a diluição que a gente encontra errada na geladeira, tal medicamento é para ser diluído em $1,8 \mathrm{ml}$, e a gente encontra com $5 \mathrm{ml}$, a gente encontra com $8 \mathrm{ml}$, e querendo ou não traz prejuízos pra criança que está internada. (T. 10)

Fica evidente que na compreensão dos participantes, o erro de medicação pode ocorrer em qualquer fase da terapia medicamentosa. Conforme literatura, esse processo é constituído pelas seguintes etapas: prescrição, distribuição ou dispensação, preparo, 


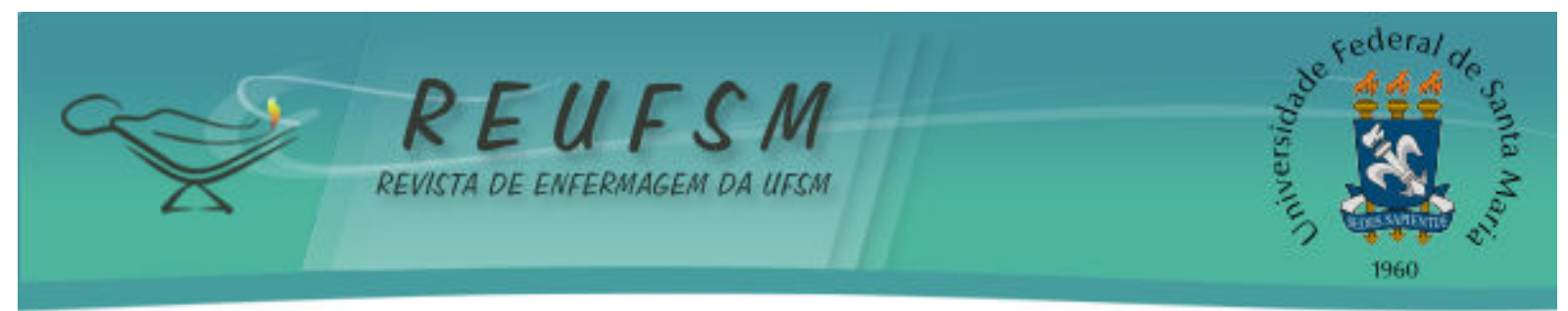

administração do medicamento e monitorização do paciente para verificação de possíveis reações medicamentosas. ${ }^{13}$

Destaca-se neste fator de risco que o erro de medicação, além da possibilidade de gerar graves consequências para os pacientes, implica na necessidade de procedimentos extras, com consequentes gastos financeiros adicionais para as instituições e aumento de horas de dedicação da Enfermagem e de outros profissionais. ${ }^{14-15}$

Além disso, a Unidade em questão apresenta a particularidade de ser um Hospital Escola, recebendo alunos de diversas áreas, com diferentes graus de aprendizado e experiência na assistência. Assim, sugere-se que há a possibilidade de uma tendência maior para a ocorrência de erros, sendo fundamental, portanto, que barreiras sejam implantadas para a prevenção e promoção de uma assistência mais segura.

[...] às vezes tu vai ler aquela prescrição e pensa nossa, mas essa aqui é uma dose muito grande... e aí... lá as vezes... o erro deles foi que eles não dividiram aquela medicação, aquela dosagem era pra ser fracionada, pra de 6 em 6 horas. (T.7)

Nesse sentido, considerando que os erros de medicação são uma grande preocupação mundial, a Aliança Mundial para Segurança do Paciente lançou o documento Soluções para a Segurança do Paciente. Entre as soluções, citam o gerenciamento de medicamentos com aparência ou com nomes semelhantes; controlar soluções eletrolíticas concentradas; e, garantir a medicação adequado em todo processo de tratamento. ${ }^{3}$

Sendo assim, todos os profissionais envolvidos na assistência devem ser comprometidos com a segurança para que os fatores de riscos relacionados à terapêutica medicamentosa sejam diminuídos e se obtenha um tratamento medicamentoso seguro em todas as etapas.

\section{Identificação do paciente}

A identificação do paciente aparece nas falas de 13 profissionais, sendo cinco enfermeiros e oito técnicos de enfermagem, justificada como um importante fator de risco, principalmente na terapia medicamentosa. Ressaltam que o maior problema na identificação do paciente vem de cunho institucional, onde a Instituição não oferece suporte adequado para a identificação correta do paciente, como a utilização de pulseiras de identificação, placas de identificação nos leitos, espaço físico reduzido, entre outros.

[...] a falta de identificação no berço, que é pouca, eu acho muito pequeno [...] muita gente, tudo muito junto, que eu acho que isso facilita um pouco. (T. 3)

[...] pela parte de identificação de leitos mesmo, o quarto coletivo, usarem cinco berços dentro de um quarto só [...]. (T. 5)

Demais estudos também evidenciam que os profissionais reconhecem que a identificação incorreta pode acarretar danos aos pacientes, sendo este um dos principais fatores que levam o profissional a cometer erros de medicação. ${ }^{16-17}$

Sendo a identificação do paciente um dos fatores de risco evidenciados pelos participantes, a literatura também mostra que a falhas na identificação geram grandes consequências para o paciente. A Organização Mundial da Saúde aponta que as principais consequências da incorreta identificação são os erros de medicação, erros de transfusão 


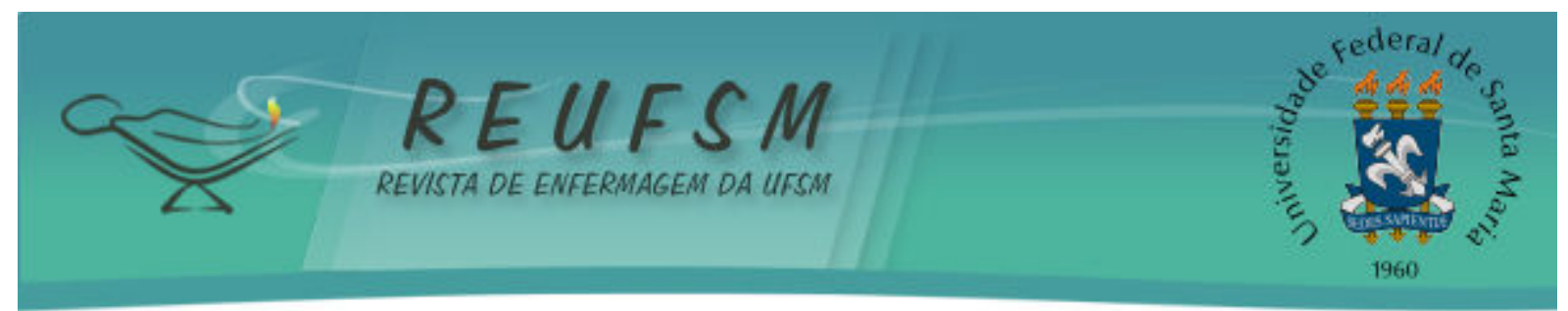

sanguínea, erros de diagnósticos, troca de pacientes na realização de procedimentos ou mesmo procedimentos em locais errados do corpo, troca de recém-nascidos, entre outros. $^{18}$

Atualmente, no Brasil, é preocupante a maneira de identificar o paciente pediátrico, visto que há poucos estudos sobre o assunto e principalmente, por ser uma área distinta, sendo fundamental o desenvolvimento de pesquisas com este enfoque. Cabe destacar, que a partir da correta identificação do paciente é possível assegurar que a assistência seja realizada da maneira certa com resultados efetivos para a segurança do paciente. A partir disso, salienta-se que é de suma importância que a instituição adote em sua política as diretrizes disponíveis na literatura quanto ao processo da correta identificação dos pacientes a fim de minimizar as possibilidades de erros assistenciais. ${ }^{19}$

\section{Métodos de barreira}

Os métodos de barreira são caracterizados por medidas preventivas que buscam diminuir os fatores de risco envolvidos durante a assistência à saúde. ${ }^{9}$ Entre as barreiras relatadas pelos participantes, evidenciaram que há lacunas de conhecimento em relação ao tema segurança do paciente, e também há uma carência de capacitações em diversos assuntos pertinentes à pediatria. Abaixo, salienta-se esta questão por meio das falas de alguns dos profissionais:

Para barreira, eu acho que começa com capacitação [...].(E.1)

[...] sempre tem que estar reciclando alguma coisa, medicação é feito, vamos lá, essa semana ou esse mês, ou de seis em seis meses, fazer seminário sobre as drogas [...]. (T. 7)

Nesse sentido, a equipe de enfermagem deve ter como meta a assistência segura e isenta de riscos ou danos aos pacientes, e para alcançá-la, esta precisa estar amparada pelo conhecimento científico, pelas normas legais que regulamentam os direitos e obrigações relativas ao exercício profissional, assim como, na dimensão ética e moral que permeiam suas ações. ${ }^{14}$ Dessa maneira, a capacitação possui a finalidade de desenvolver na equipe de Enfermagem novos saberes, habilidades e atitudes. ${ }^{20}$

Para tanto, o processo de educação continuada é fundamental para alcançar melhores resultados na qualidade da assistência. ${ }^{21} \mathrm{~A}$ atualização constante do profissional proporciona que o mesmo realize uma prática fundamentada cientificamente, sendo este um fator que determina melhores resultados assistência. ${ }^{22}$

Também abordam a questão específica da capacitação quanto ao processo de medicação, conforme falas expostas abaixo, quando afirmam a necessidade de "cinco certezas da medicação":

[...] como barreira, primeiro é ter as cinco certezas né? [...]. (T. 9)

[...] qualquer miligrama, dose errada na criança pode levar a um problema sério, diferente do adulto né, então acho que as pessoas... tá faltando isso mesmo, capacitação [...]. (T. 8)

Fica evidente a preocupação dos participantes quanto à capacitação profissional no processo de medicação. Conforme a literatura, atualmente, preconiza-se a confirmação de nove certezas da medicação, ao invés das cinco certezas apontadas nas falas acima, 


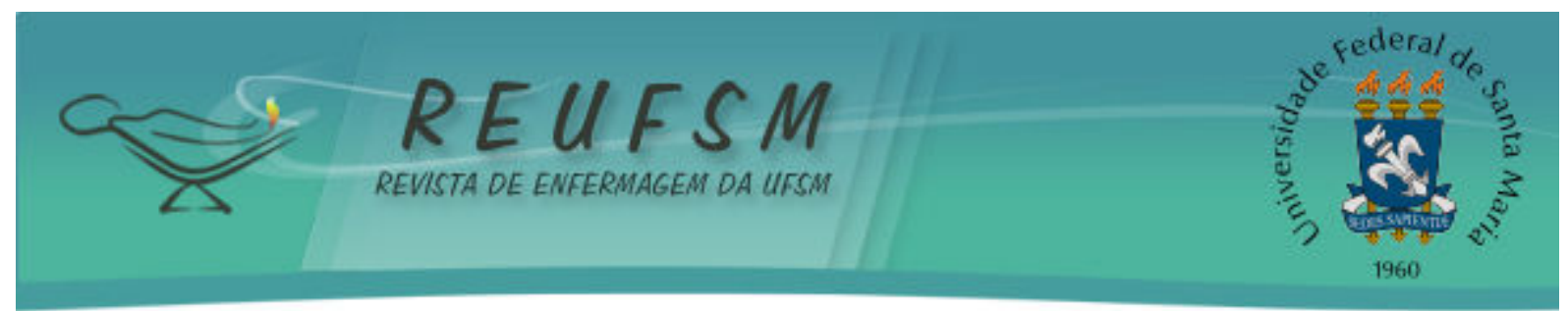

sendo elas: paciente certo, medicação certa, via certa, dose certa, hora certa, registro correto, conhecer a ação, apresentação farmacêutica e monitorar o efeito. ${ }^{23}$

Além desta medida de segurança, estudos apontam demais estratégias que devem ser consideradas durante a prática da enfermagem, tais como: prescrições informatizadas, dose unitária de medicamentos, uso de códigos de barras, bombas de infusão inteligentes, identificação do paciente e dupla checagem. Também descrevem a utilização de prescrição médica com aprazamento contemplando horário, via de administração, dose escrita de forma clara, e ainda, cuidados relativos à administração e monitorização. ${ }^{24}$

Além disso, a questão da correta identificação como um método de barreira demonstrou ser bem clara para os entrevistados. Os participantes falaram em estabelecer uma melhor identificação dos leitos e boxes de medicação com identificação individualizada, conforme na seguinte fala:

[...] de repente fazer boxes separadinhos, daí colocar número de leito mesmo e os pacientes serem alocados em cada número de leito [...]. (E. 5)

Cabe destacar que para assegurar uma correta identificação do paciente a Aliança Mundial para Segurança do Paciente preconiza nas Soluções para Segurança do Paciente este tema especificadamente para paciente pediátricos, incluindo a responsabilização dos profissionais na confirmação da identidade do paciente antes de qualquer procedimento; e ainda, utilização de três identificadores para pediatria: nome completo da criança, data nascimento e nome da mãe ou responsável da criança. Também, abordam a adoção do uso de pulseira de identificação, identificação correta e completa no prontuário e documentos, e participação ativa da criança e familiar no processo da sua identificação. ${ }^{18,25}$

Por fim, citam como mais um método de barreira a implementação de uma cultura de segurança do paciente na unidade. Surge a afirmação da equipe sobre a necessidade de iniciativa dos funcionários para uma assistência segura, baseada na notificação dos erros, preceitos estes, que são difundidas por meio da cultura de segurança do paciente:

[...] primeiro tem que partir de uma cultura de segurança da unidade, [...] tem que ter um movimento de que toda a equipe esteja ciente e envolvida no sentido de promover a cultura de segurança do paciente, [...] acho que a primeira coisa é a conscientização da equipe né? [...]. (E. 2)

[...] eu acho também que uma outra medida que a gente precisa ter é fazer a notificação do erro [...]. (E. 2)

Conforme resultados de estudos, a adoção de uma cultura de segurança do paciente favorece a notificação dos erros, sendo possível determinar as causas de origem. Assim, ações específicas podem ser planejadas para evitar a recorrência dos erros. ${ }^{26}$ Estudo realizado em um Hospital espanhol revelou que após utilizarem estratégias educativas com foco na cultura de segurança, houve uma redução significativa dos erros relacionados à prescrição de medicamentos, caindo de $21 \%$ para $3 \%{ }^{27}$

Neste contexto, os profissionais e a organização de saúde podem adotar medidas a fim de estabelecer a cultura de segurança do paciente nas instituições e fortalecer a prática de hospitalizações mais seguras. 


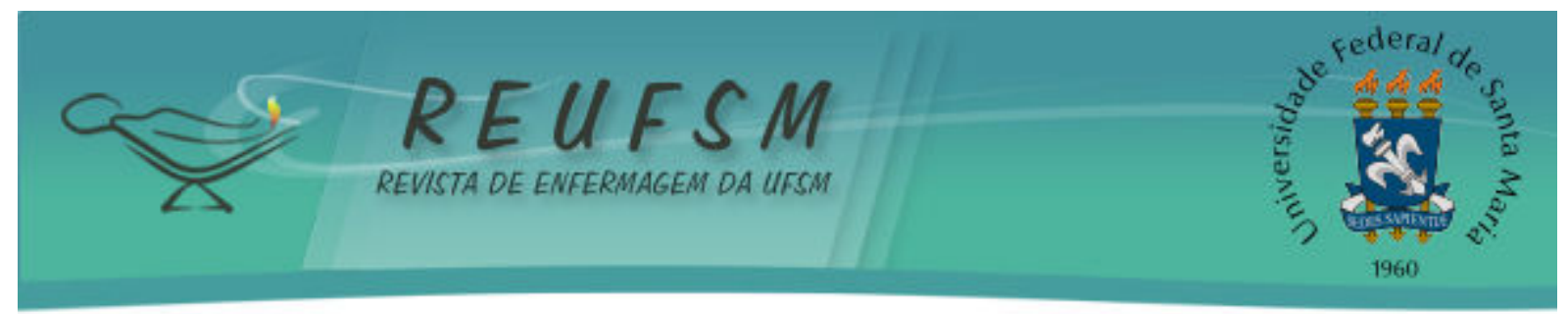

CONCLUSÃO

0 presente estudo possibilitou conhecer a percepção da equipe de enfermagem acerca dos fatores de risco que podem interferir na segurança do paciente pediátrico durante sua hospitalização, assim como identificar sua percepção relacionada aos métodos de barreiras para garantir a segurança destes pacientes.

Os resultados evidenciam que a percepção dos enfermeiros relacionada aos fatores de risco foi embasada em um conhecimento científico prévio, de acordo com os preceitos da OMS, no entanto, não foi expressa uma interligação deste termo com o conceito oficial. Já, o conhecimento dos técnicos e auxiliares de enfermagem representou ser fruto da prática cotidiana, pois identificaram como fatores de risco à segurança do paciente as ações do processo assistencial realizado na unidade, como segurança na medicação, identificação do paciente e risco de quedas.

Sendo assim, os resultados mostraram que os membros da equipe de enfermagem convergem em suas percepções, pois identificaram alguns fatores de risco, às vezes evidenciados pelo cotidiano, não relacionando com o conceito da OMS.

Além disso, citaram como métodos de barreiras a capacitação profissional por meio da educação continuada, a correta identificação do paciente, sistema de notificação de eventos adversos e adoção de uma cultura de segurança.

A partir disso, ressalta-se a necessidade em qualificar os profissionais de enfermagem constantemente, por meio de capacitações e cursos, sobre a temática segurança do paciente. Para tanto, faz-se necessária à sensibilização dos profissionais para o desenvolvimento de estratégias que amplifiquem os olhares sobre os fatores de risco e segurança do paciente, e implantem barreiras de segurança, trazendo como resultados uma assistência segura, tanto para a saúde das crianças hospitalizadas quanto para agregar qualidade à assistência prestada.

Por fim, recomenda-se que demais pesquisas sejam realizadas com foco nestas questões de segurança do paciente pediátrico a fim de proporcionar subsídios para a prática assistencial segura, buscando identificar os problema e sensibilizar os profissionais sobre os fatores de risco que podem interferir na segurança do paciente.

\section{REFERÊNCIAS}

1. Silva LD. Segurança do paciente no contexto hospitalar. Rev Enferm UERJ. 2012 jul/set; 20(3):291-2.

2. Organização Mundial da Saúde (OMS). Learning from error. Patient Safety Workshop [Internet]. 2010 [acesso em 2012 maio 5]. Disponível em: http://www.who.int/patientsafety/education/vincristine_download/en/index.html.

3. Organização Mundial da Saúde (OMS). Organização Pan Americana da Saúde (OPAS). Aliança Mundial para Segurança do Paciente [Internet]. 2010 [acesso em 2012 maio 5]. Disponível em: http: //new.:paho.org/bra/index.php?option=com_content\&task=view\&id=931\&ltemid=686.

4. Corbellini VL, Schilling MCL, Frantz SF, Godinho TG, Urbanetto JS. Eventos adversos relacionados a medicamentos: percepção de técnicos e auxiliares de enfermagem. Rev Bras Enferm. 2011;64(2):241-7.

5. National Patient Safety Agency. Review of patient safety for children and young people. London: National Patient Safety Agency; 2009. 


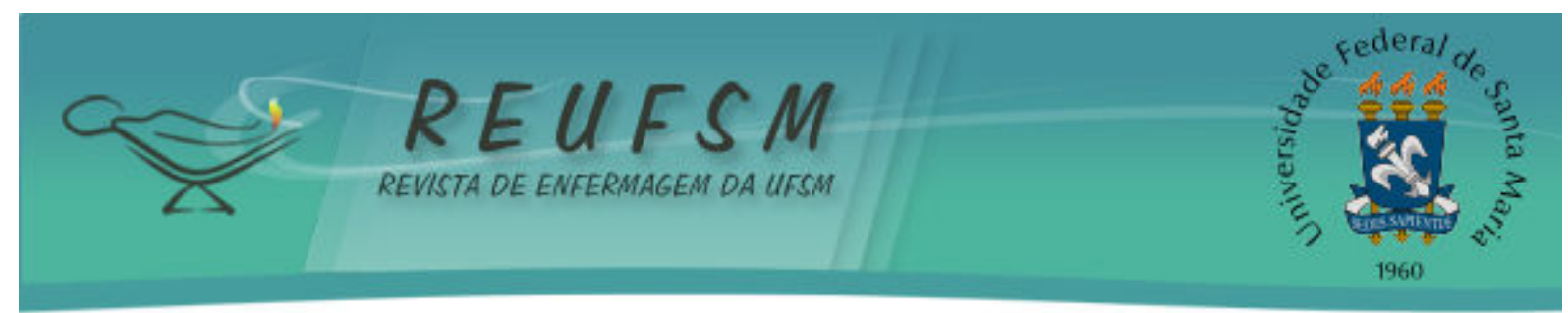

6. Belela AS, Peterlini MA, Pedreira ML. Revelação da ocorrência de erro de medicação em unidade de cuidados intensivos pediátricos. Rev Bras Ter Intensiva. 2010;22(3):257-63.

7. Kashual R, Bates DW, Landrigan C, Mckenna KJ, Clapp MD, Federico F, et al. Errors and adverse drug events in pediatric inpatients. Am Med Assoc. 2001;285(16):2114-20.

8. Yamamoto MS, Peterlini MAS, Bohomol E. Notificação espontânea de erros de medicação em hospital universitário pediátrico. Acta Paul Enferm [Internet]. 2011 [acesso em 2012 maio 20];24(6):766-71.

http://www.scielo.br/pdf/ape/v24n6/a06v24n6.pdf.

Disponível

em:

9. Santana JCB, Sousa MA, Soares HC, Avelino KSA. Fatores que influenciam e minimizam os erros na administração de medicamentos pela equipe de enfermagem. Enferm Rev [Internet]. 2012 [acesso em 2012 dez 5];15(1):122-37. Disponível em: http://periodicos.pucminas.br/index.php/enfermagemrevista/article/view/3300.

10. Minayo MCS. O desafio do conhecimento: pesquisa qualitativa em saúde. 12a ed. São Paulo: Hucitec; 2010.

11. Jirapaet V, Jirapaet K, Sopajaree C. The nurses experience of barriers to safe practice in the neonatal intensive care unit in Thailand. JOGNN; 2006. p. 746-54.

12. Peterlini MAS, Pereira SR. Os erros humanos: abrangência e tipos. In: Harada MJCS, Pedreira MLG, Peterlini MAS, Pereira SR, organizadores. $O$ erro humano e a segurança do paciente. São Paulo: Atheneu; 2006. p. 19-26.

13. Harada MJCS, Chanes DC, Kusahara DM, Pedreira MLG. Segurança na administração de medicamentos em Pediatria. Acta Paul Enfermagem [Internet]. 2012 [acesso em 2012 maio 11];25(4):639-42. Disponível em: http://www.scielo.br/pdf/ape/v25n4/25.pdf.

14. Santos JO, Silva AEBC, Munari DB, Miasso Al. Condutas adotadas por técnicos de enfermagem após ocorrência de erros de medicação. Acta Paul Enfermagem [Internet]. 2010 [acesso em 2012 set 18];23(3):328-33. Disponível em: http://dx.doi.org/10.1590/S0103-21002010000300003.

15. Fassini $P$, Hahn GV. Riscos à segurança do paciente em unidade de internação hospitalar: concepções da equipe de enfermagem. Rev Enferm UFSM [Internet]. 2012 [acesso em 2012 nov 26];2(2):290-9. Disponível em: http://cascavel.ufsm.br/revistas/ojs2.2.2/index.php/reufsm/article/view/4966/3753.

16. Wegner W, Pedro ENR. A segurança do paciente nas circunstâncias de cuidado: prevenção de eventos adversos na hospitalização infantil. Rev Latinoam Enferm. 2012 [acesso em 27/01/2014];20(3):[8 telas]. Disponível em: http: / / www.scielo.br/scielo.php?script=sci_arttext\&pid=S0104$11692012000300002 \&$ lng=pt\&nrm=iso\&tlng=pt.

17. Corbellini VL, Schilling MCL, Frantz SF, Godinho TG, Urbanetto JS. Eventos adversos relacionados a medicamentos: percepção de técnicos e auxiliares de enfermagem. Rev Bras Enferm. 2011 mar-abr;64(2):241-7.

18. World Health Organization (WHO). Patient Safety Solutions [Internet]. 2007 [acesso em 2012 ago 15]. (Vol. 1, Solution 2). Available from: http://www.who.int/patientsafety/solutions/patientsafety/PS-Solution2.pdf.

19. Porto TP, Rocha PK, Lessmann JC, Souza S, Kretzer L, Anders JC. Identificação do Paciente em Unidade Pediátrica: uma questão de segurança. Rev Soc Bras Ped. 2011;11(2):67-74. 


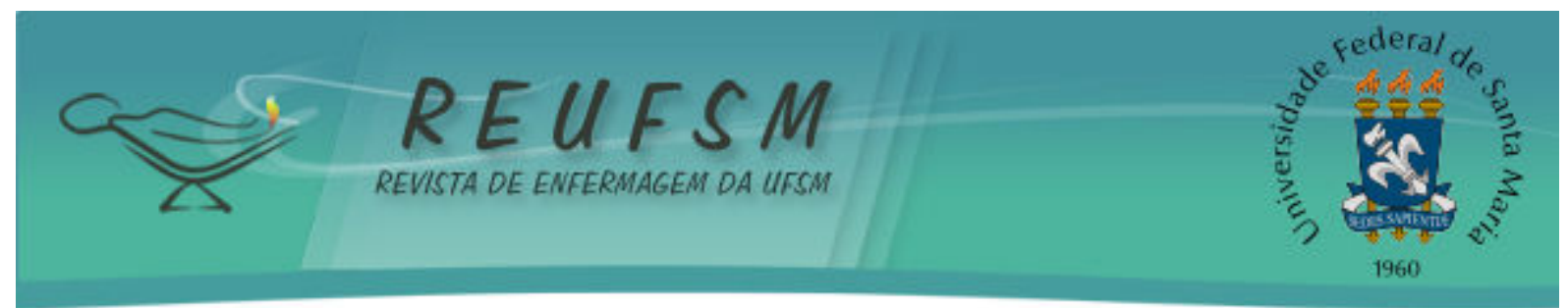

20. Franciscatto L, Bessow CK, Ruzczyk JVA, Oliveira MA, Kluck MM. Metas internacionais de segurança do paciente em hospital universitário. Rev HCPA. 2011;31(4):482-6.

21. Silva RCL, Cunha JJSA, Moreira CLS. Evento Adverso em Terapia Intensiva: O que sabem os profissionais de enfermagem. Rev Cuid Fundam [Internet]. 2011 [acesso em 2012 maio 18];3(2):1848-55. Disponível em: http://www.seer.unirio.br/index.php/cuidadofundamental/article/view/1393/pdf_384.

22. Telles Filho PCP, Praxedes MFS, Pinheiro MLP. Erros de medicação: análise do conhecimento da equipe de enfermagem de uma instituição hospitalar. Rev Gaúch Enferm. 2011 set;32(3):539-45.

23. Costa DB, Vannuchi MTO, Haddad MCFL, Cardoso MGP, Silva LG, Garcia SD. Custo de educação continuada para equipe de enfermagem de um hospital universitário público. Rev Eletrônica Enferm [Internet]. 2012 [acesso em 2012 jun 2];14(2):257-66. Disponível em: http://dx.doi.org/10.5216/ree.v14i2.14540.

24. Silva LD, Camerini FG. Análise da administração de medicamentos intravenosos em hospital da rede sentinela. Texto \& Contexto Enferm [Internet]. 2012 [acesso em 2012 jun 16];21(3):633-41. Disponível em: http://www.scielo.br/pdf/tce/v21n3/v21n3a19.pdf.

25. Conselho Regional de Enfermagem do Estado de São Paulo - Coren-SP. 10 Passos para a segurança do paciente [Internet]. 2010 [acesso em 2013 fev 20]. Disponível em: http://www.corensp.org.br/node/34764.

26. Lage MJ. Segurança do doente: da teoria à prática clínica. Rev Port Saúde Pública. 2012;10:11-6.

27. Campino A, Lopez-Herrera MC, Lopez-de-Heredia I, Valls-i-Soler A. Educational strategy to reduce medication errors in a neonatal intensive care unit. Acta Pædiatrica. 2009;98:782-85.

Data de recebimento: $24 / 04 / 2013$

Data de aceite: $27 / 01 / 2014$

Contato com autor responsável: Patrícia Kuerten Rocha

Endereço: Av Buriti, 620, apto 508, bloco B, Itacorubi, Florianópolis. Santa Catarina, Brasil,CEP: 88034500

Email: pkrochaucip@gmail.com 